\title{
Conference presentation
}

\section{Comparison of effects of homeopathic medicines prepared in glass and plastic vials in macrophage activity in vitro}

Luciana C Dalboni [1], Fabiana R Santana [1], Thayná N Cardoso [1], Fabiana Toshie Konno [1], Silvia Waisse Priven [2], Amarilys T Cesar [3], Leoni V Bonamin [1]

[1] Universidade Paulista, UNIP, Brazil, [2] Pontifícia Universidade Católica de São Paulo, PUC-SP, Brazil, [3] Farmácia NH Cristiano, São Paulo, Brazil

Background: According to the "silica hypothesis" to explain the action of homeopathic medicines, complex nanoparticles involving the silica extracted from the walls of the glass vial during succussions would be needed to act on target cells. Aims: We aimed to know if suspended particles of silica found in Arsenicum album (AA) could be associated to its biological effects in vitro. Methodology: RAW 264.7 macrophages were co-cultured with yeast (Saccharomyces cerevisiae) to promote phagocytosis. During this process, the cells were exposed to AA in different homeopathic dilutions $(30 \mathrm{~K}, 6 \mathrm{cH}$ and $200 \mathrm{cH})$ prepared in both, glass or silicafee plastic vials. All dilutions were prepared from the same $3 \mathrm{cH}$ matrix. Since parallel experiments showed random stimulating and inhibiting effects of homeopathic lactose, untreated cells and water were used as controls. The macrophage activity was measured by digital histometry (Metamorph $(\AA)$ ), reflecting the cell spreading and phagocytosis. A complementary analysis using the acridine orange stain in real time fluorescence microscopy was also performed to evaluate the organization of phago-lysosomes inside the cells. All experiments were performed in triplicate. Microparticles present in the medicines were identified in an EDS (energy dispersive x-ray detector) system (JEOL JMS 6510), according to their chemical elements composition. The morphology of particles was not considered for analysis, since it was considered irrelevant. Results: Experment one: AA 30K and pure water, when prepared in plastic vials, were able to increase macrophage activity $(\mathrm{p} \leq 0.05)$, but the same result was not seen in cells treated with AA $30 \mathrm{~K}$ or pure water stocked in glass. Experiment two: AA 200cH prepared in plastic vials allso le ad o signif capt increase of macrophage spreading $(\mathrm{p} \leq 0.05)$ in relation to the controls, but $\mathrm{n}$ g ffect wass en in the samples prepared in glass vials. Experiment three: No difference was observed in AA $6 \mathrm{cH}$ treated cells in relation to the controls. The morphology of the cells under fluorescence microscopy exhibited increase in lysosome activity in AA $200 \mathrm{cH}$ prepared in both plastic and glass vials, in relation to the untreated control. The EDS analysis has shown that only medicines prepared in glass vials contained suspended silica particles. Conclusions: Taken the data together, the unspecific effects on macrophage spreading after treatment with homeopathic AA $30 \mathrm{~K}, 200 \mathrm{cH}$ and water prepared in plastic vials, added to the absence of silica particles in these preparations and the increase of lysosomal activity only seen in AA $200 \mathrm{cH}$, independently of the vial nature, corroborate that silica might not be a mandatory condition to justify the biological effects of homeopathic medicines. Additionally, the existence of putative contaminants released from plastic vials during the medicine manipulation deserves further studies.

Acknowledgements: CAPES, UNIP, ABFH

(C) International Journal of High Dilution Research. Not for commercial purposes. 